\title{
Palbociclib as an early-line treatment for Japanese patients with hormone receptor-positive/human epidermal growth factor receptor 2-negative advanced breast cancer: a review of clinical trial and real-world data
}

\author{
Norikazu Masuda ${ }^{1}$ (D) $\cdot$ Nobuyoshi Kosaka $^{2} \cdot$ Hiroji Iwata $^{3} \cdot$ Masakazu Toi $^{4}$
}

Received: 24 May 2021 / Accepted: 19 August 2021 / Published online: 26 October 2021

(c) Pfizer Inc. 2021

\begin{abstract}
Breast cancer is the most common type of cancer among women worldwide and in Japan. The majority of breast cancers are hormone receptor-positive (HR+)/human epidermal growth factor receptor 2-negative (HER2-), and endocrine therapy is an effective therapy for this type of breast cancer. However, recent substantial advances have been made in the management of HR+/HER2 - advanced breast cancer (ABC) with the advent of targeted therapies, such as cyclin-dependent kinase 4/6 (CDK4/6) inhibitors, resulting in significant improvements in survival outcomes versus endocrine therapy alone. To evaluate the optimal use of palbociclib, a CDK4/6 inhibitor, in HR+/HER2- ABC, this review summarizes clinical trial and realworld data for palbociclib. In addition, current biomarker studies in palbociclib clinical research are reviewed. In Japanese patients, palbociclib was shown to be effective with a manageable safety profile, although differences were observed in the frequency of adverse event and dosing parameters. Current evidence supporting palbociclib as a first-line treatment strategy for patients with HR+/HER2- ABC in Asia, and specifically japan, is also discussed.
\end{abstract}

Keywords Advanced breast cancer $\cdot$ Clinical trial $\cdot$ HR+/HER2 $-\cdot$ Palbociclib $\cdot$ Real-world

\section{Introduction}

Breast cancer is the most common cancer among women worldwide and also in Japan [1] and is the fifth leading cause of cancer-related death in Japanese women [2]. In eastern Asia (inclusive of Japan) in 2018, the incidence of breast cancer was 39.2 per 100,000 females, whereas the mortality rate was 8.6 per 100,000 females [1]. Four main molecular subtypes of breast cancer exist (i.e., hormone receptor-positive/human epidermal growth factor receptor 2-negative [HR+/HER2-], HR+/HER2+, HR-/HER2-, and

Norikazu Masuda

nmasuda@alpha.ocn.ne.jp

1 National Hospital Organization Osaka National Hospital, 2-1-14 Hoenzaka, Chuou-ku, Osaka-city 540-0006, Japan

2 Pfizer Japan Inc, Tokyo, Japan

3 Aichi Cancer Center Hospital, Aichi, Japan

4 Graduate School of Medicine, Kyoto University, Kyoto, Japan
HR-/HER2+) [3]. Among patients diagnosed with primary breast cancer, the majority $(71-73 \%)$ of breast cancers are HR+/HER2- [3, 4].

Monotherapy with an aromatase inhibitor or fulvestrant is a reasonable treatment option for $\mathrm{HR}+$ advanced breast cancer (ABC) considering the economic and clinical benefit to patients. A previous study in patients with estrogen receptor-positive $(\mathrm{ER}+) \mathrm{ABC}$ or metastatic breast cancer (MBC) reported a median overall survival (OS) of 54.1 months with fulvestrant and 48.4 months with anastrozole [5]. In Japanese patients with $\mathrm{HR}+\mathrm{ABC}$ who were treated with anastrozole, the median time to progression was 13.7 months, and median OS was 60.1 months [6]. However, it is essential to consider how OS can be extended beyond 5 years in patients with $\mathrm{ABC}$, since data suggest that initial treatment of $\mathrm{ABC}$ with chemotherapy does not provide a survival advantage over endocrine therapy [7].

Cyclin-dependent kinase 4/6 (CDK4/6) and cyclin D1 together play a role in regulating cell-cycle progression (Fig. 1) [8, 9]. Palbociclib was the first CDK4/6 inhibitor approved for the treatment of $\mathrm{HR}+/ \mathrm{HER} 2-\mathrm{ABC}$ in 
0
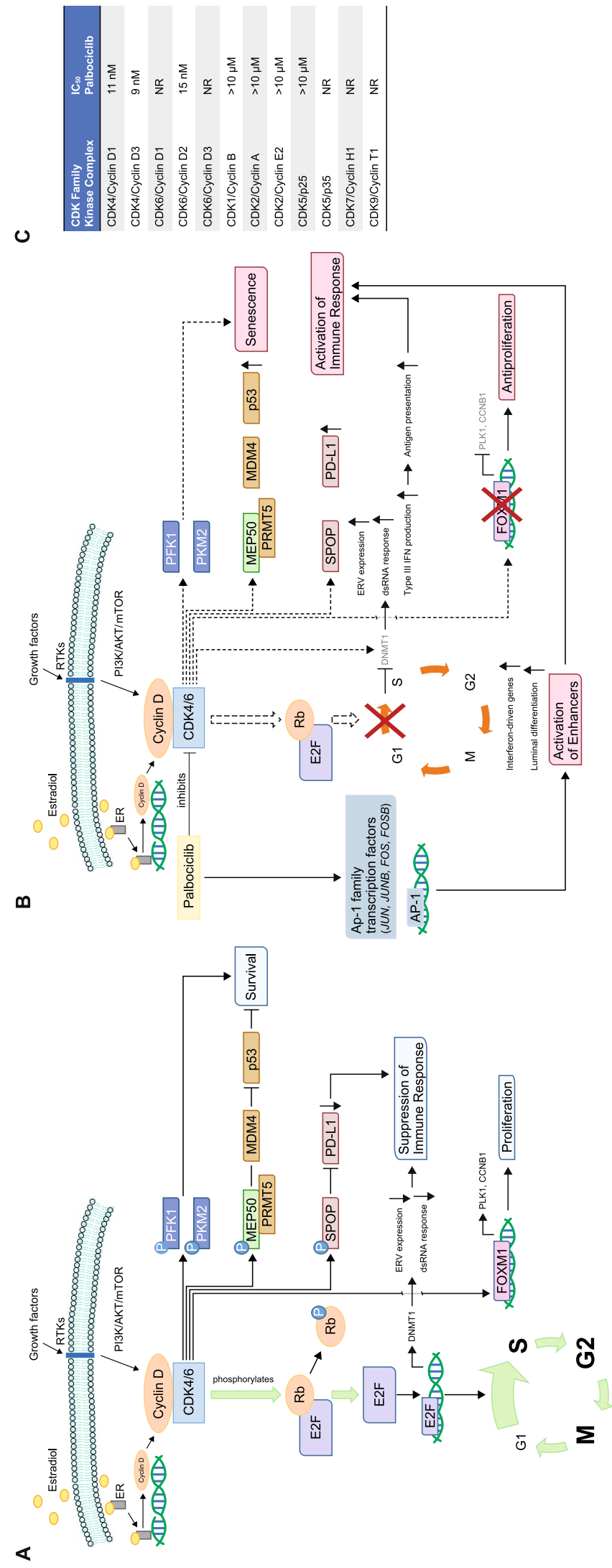

至 $\overline{\mathrm{d}}$

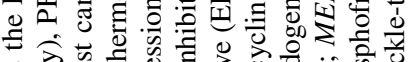

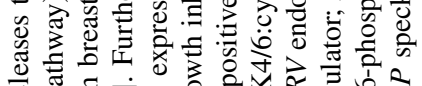

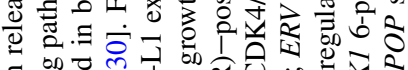

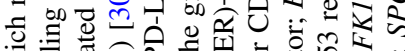

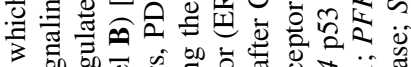

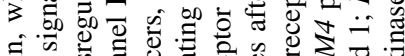

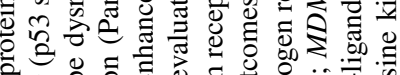

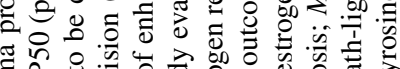

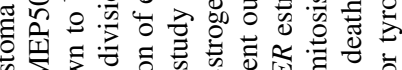

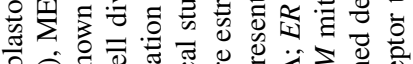

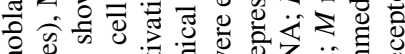

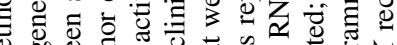

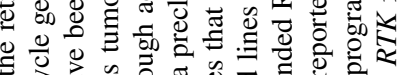

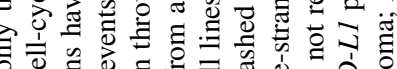

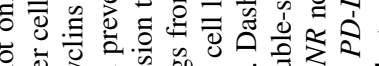

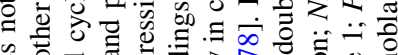

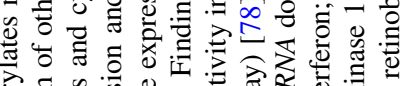

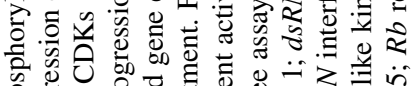

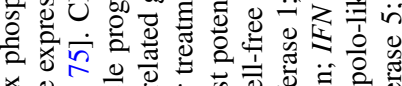

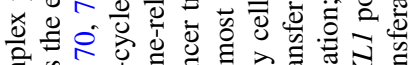

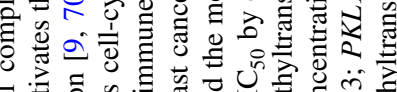
$\bar{a}$ 密.

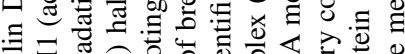
递恶

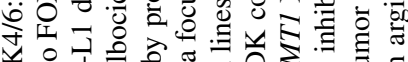
光品完灾。

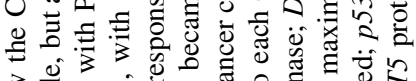

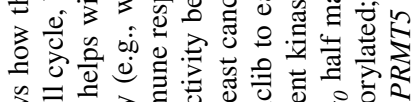

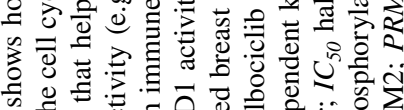

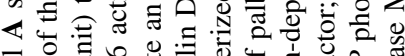

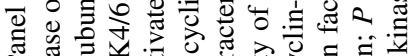
记 की

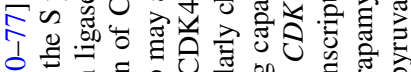
르을

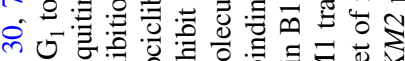
人。

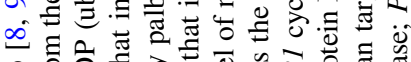

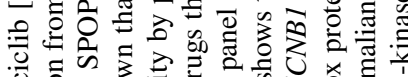
U.

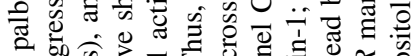

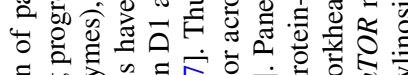

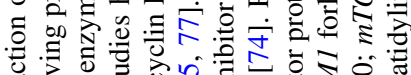

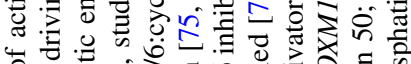

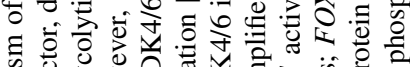

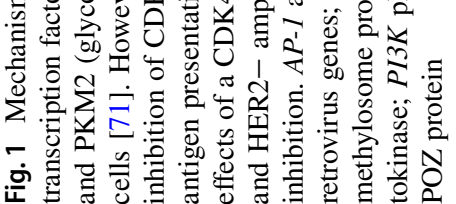


combination with an aromatase inhibitor in the first-line setting or fulvestrant in the second-line setting in the United States [10]. In Japan, palbociclib was approved for the treatment of inoperable or recurrent breast cancer in 2017 [11]. Current Japanese Breast Cancer Society Clinical Practice guidelines recommend a CDK4/6 inhibitor plus an aromatase inhibitor as a first-line endocrine therapy for postmenopausal patients with HR+/HER2- ABC [12].

Early preclinical studies showed that palbociclib was a potent and highly selective CDK4/6 inhibitor, and that targeting CDK4/6 alone resulted in antitumor activity [13, 14]. Two other CDK $4 / 6$ inhibitors, ribociclib and abemaciclib, have also been developed for the treatment of HR+/HER2- ABC [15]. The clinical benefits of palbociclib $[16,17]$, ribociclib [18, 19], and abemaciclib [20, 21] have been shown in randomized clinical trials, with all 3 CDK4/6 inhibitors showing comparable efficacy results and manageable safety profiles without a deterioration in quality of life [22]. The focus of this review is to summarize current clinical trial data and real-world evidence supporting palbociclib as the first-line standard of care for HR+/HER2- ABC.

\section{Review of palbociclib clinical trial data}

\section{Palbociclib clinical trials}

The phase 3 PALOMA-2 and PALOMA-3 trials showed significant improvement in PFS with palbociclib plus letrozole or fulvestrant versus placebo plus letrozole or fulvestrant, respectively (Table 1) [16, 17, 23-25]. In PALOMA-2, postmenopausal women with ER+/HER2- ABC were randomized 2:1 to receive palbociclib or placebo plus letrozole as first-line treatment for their advanced disease [16, 23]. The median PFS was 27.6 months in the palbociclib group versus 14.5 months in the placebo group. Furthermore, a subgroup analysis of patients enrolled in PALOMA-2 showed a PFS benefit with palbociclib plus letrozole versus placebo plus letrozole across all patient subgroups, including among patients with bone-only disease [23]. Moreover, median PFS was significantly longer in the palbociclib group compared with the placebo group among patients with visceral metastases (19.3 months [95\% CI, 16.4-22.2] vs 12.9 months [8.4-16.6], respectively; hazard ratio $=0.63$ [95\% CI, 0.47-0.85]; $P<0.01$ ), and among patients without visceral metastases (not reached [95\% CI, 25.1-not estimable] vs 16.8 months [95\% CI, 13.7-22.2]; hazard ratio $=0.50$ [95\% CI, 0.36-0.70]; $P<0.0001$ ) [26]. In PALOMA-3, pre/perimenopausal and postmenopausal women with HR+/HER2- ABC whose cancer had relapsed or progressed with prior endocrine therapy were randomized 2:1 to receive palbociclib or placebo plus fulvestrant $[17,24]$. Median PFS was 11.2 and 4.6 months in the palbociclib and placebo groups, respectively. Moreover, OS analysis from PALOMA-3 after 44.8 months of follow-up showed an OS benefit of 6.9 months with palbociclib plus fulvestrant versus placebo plus fulvestrant [25]. Overall survival data from PALOMA-2 have not yet been reported.

In both phase 3 PALOMA trials, neutropenia was the most common grade 3 or 4 adverse event (AE) [16, 17]. Among patients in the palbociclib plus letrozole group in PALOMA-2, 56.1\% experienced grade 3 neutropenia and $10.4 \%$ experienced grade 4 neutropenia; the incidence of grade 3 or 4 febrile neutropenia was low (1.8\%) [16]. In PALOMA-3, among patients in the palbociclib plus fulvestrant group, $53.3 \%$ experienced grade 3 and $8.7 \%$ experienced grade 4 neutropenia; febrile neutropenia was reported in $0.6 \%$ of patients [17]. A pooled analysis of data from the PALOMA-1, -2 , and -3 trials showed that interstitial lung disease was reported in $1.5 \%$ of patients receiving palbociclib plus endocrine therapy and that the incidence of interstitial lung disease was similar across geographic locations [27]. Additionally, quality of life was maintained in patients treated with palbociclib plus endocrine therapy $[28,29]$.

Subsequent treatments after permanent discontinuation of palbociclib were also assessed in both phase 3 trials (Table 1). These findings indicate that palbociclib did not compromise the efficacy of subsequent therapy, and that palbociclib combination therapy extended the time to chemotherapy [23, 25]. Moreover, the types of subsequent therapies patients received were generally similar between treatment arms in both PALOMA-2 and PALOMA-3, suggesting that palbociclib does not influence the subsequent therapy received [23, 25].

Biomarker analyses using patient tumor samples were conducted in both PALOMA-2 and PALOMA-3. Data from PALOMA-2 reinforced ER status as a significant marker for therapeutic benefit with observations supporting that this breast cancer subtype is dependent on the CDK4/6:cyclin D:retinoblastoma pathway [30]. In addition, higher levels of $C D K 4$ gene expression were suggestive of an endocrine resistance phenotype that could be circumvented with the addition of palbociclib (Fig. 2) [30]. Analyses of PALOMA-3 showed that lower levels of CCNE1 mRNA expression were linked to greater PFS benefit with palbociclib plus fulvestrant treatment [31]. Analyses of circulating tumor DNA (ctDNA) from PALOMA-3 showed that with both palbociclib plus fulvestrant and placebo plus fulvestrant treatment, TP53 mutation and FGFRI gain were associated with early relapse [32]. Moreover, PIK3CA ctDNA dynamics after 2 weeks of palbociclib plus fulvestrant treatment were predictive of long-term outcomes [33].

Additionally, an analysis was performed based on whole-exome sequencing of 59 tumors from patients with HR+/HER2- MBC who received CDK4/6 inhibitors to evaluate mechanisms driving resistance to CDK4/6 


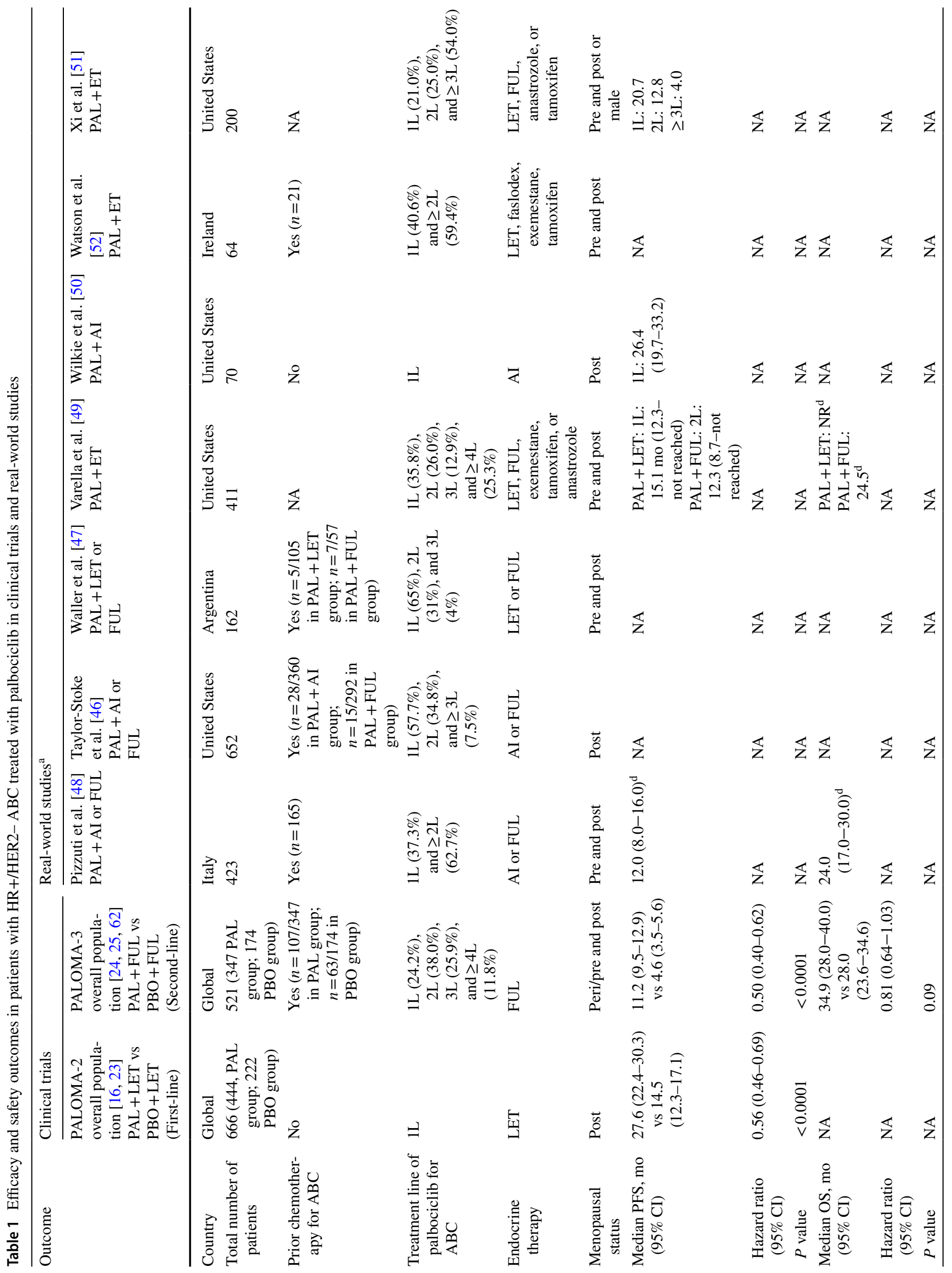




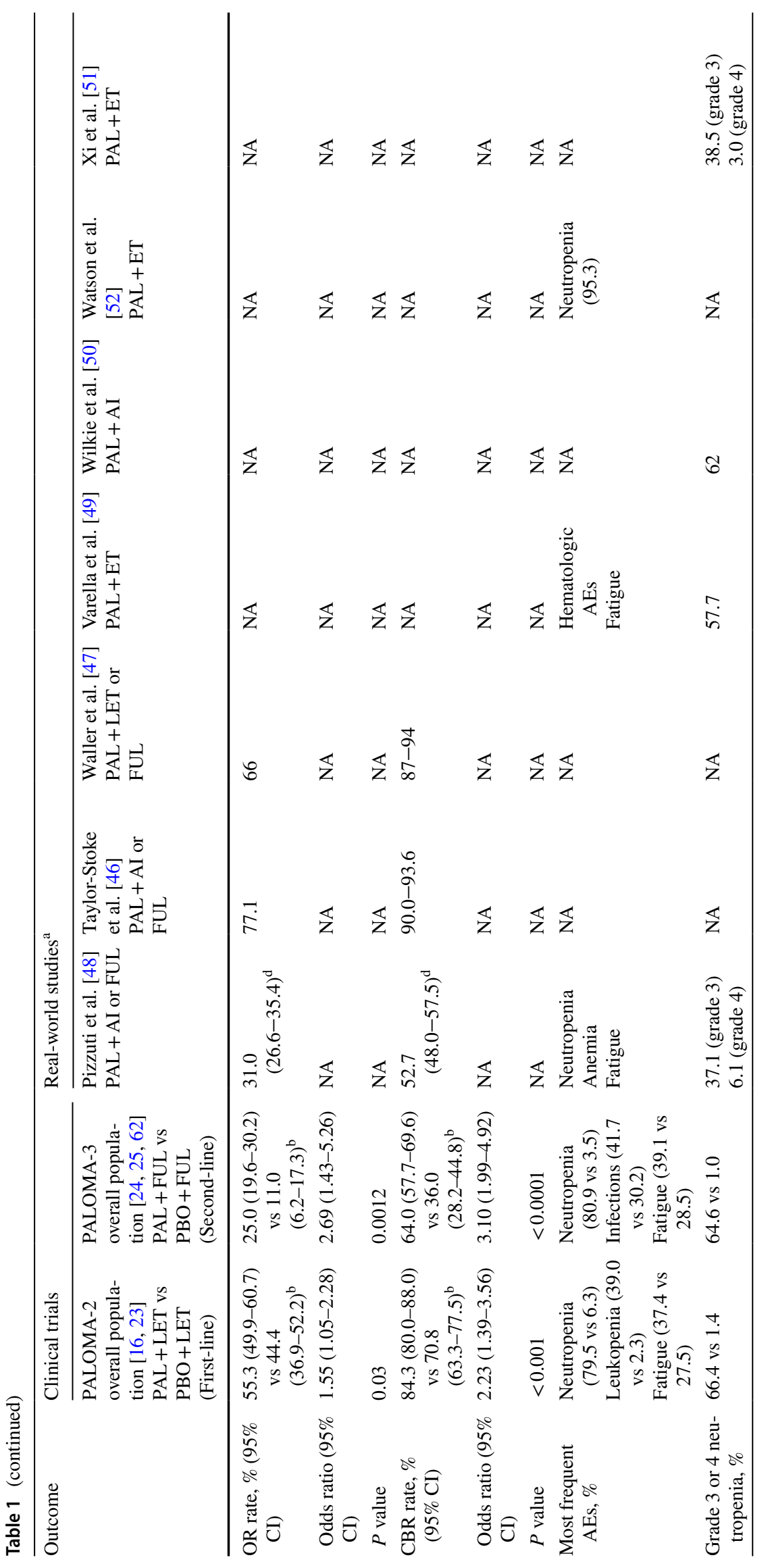




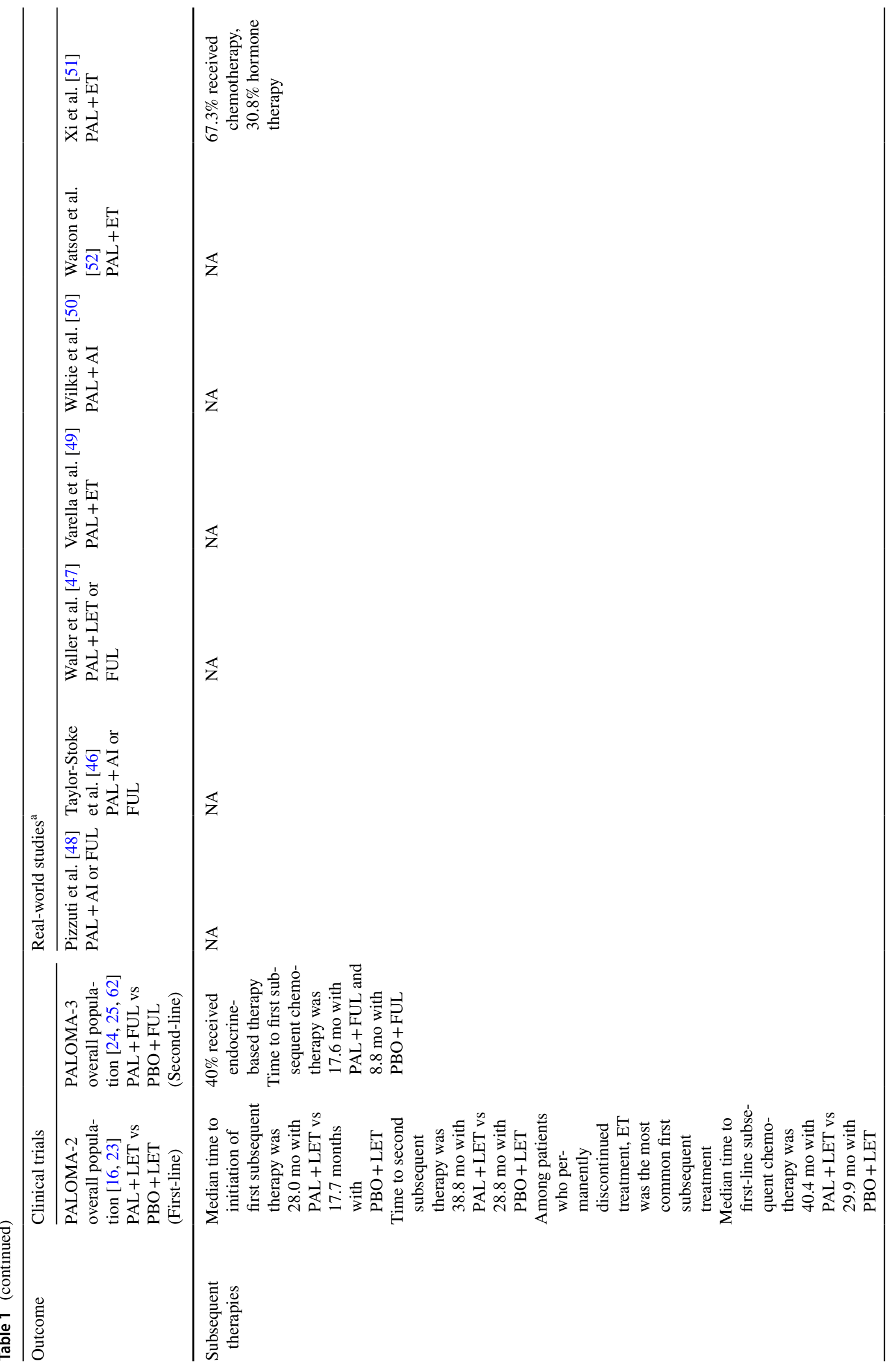




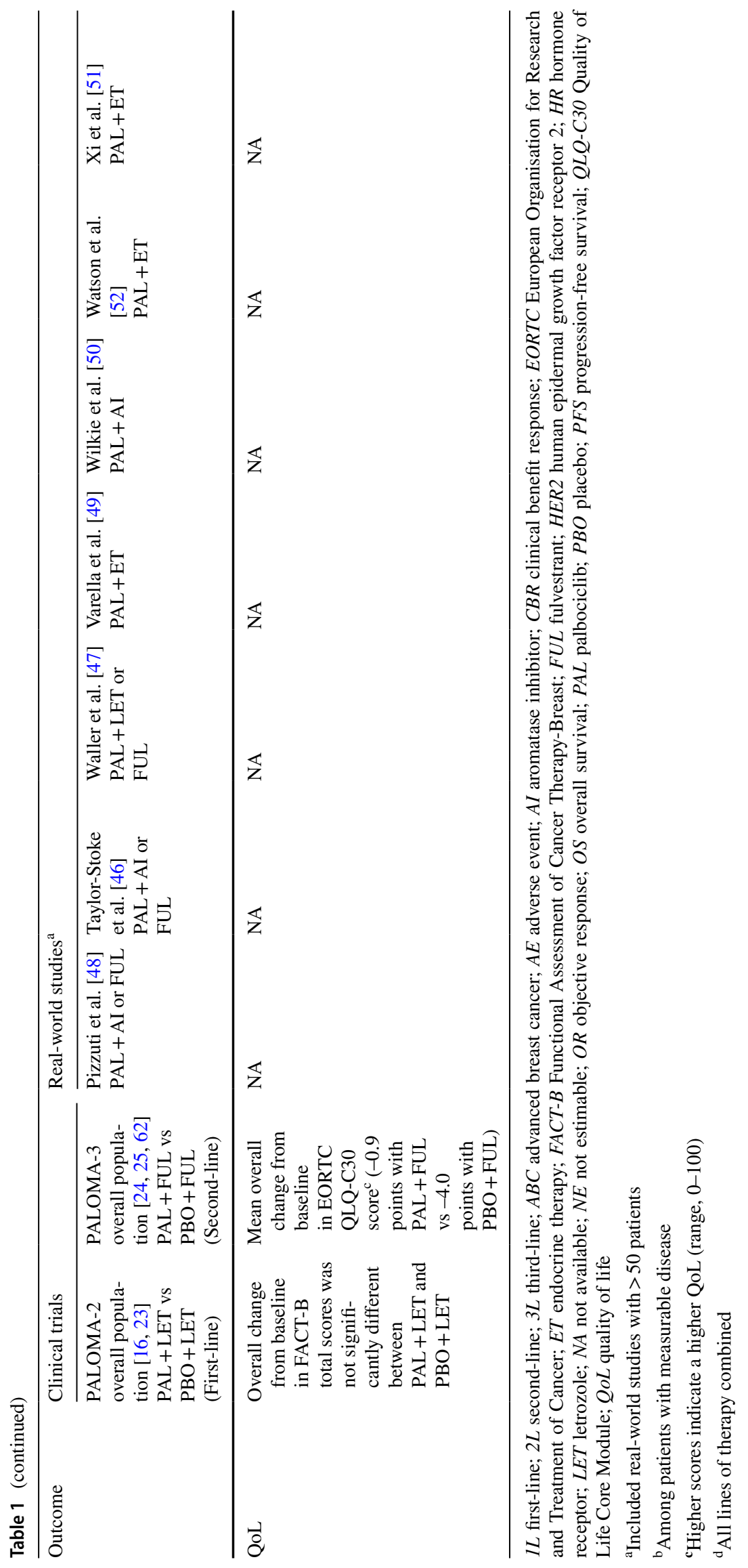




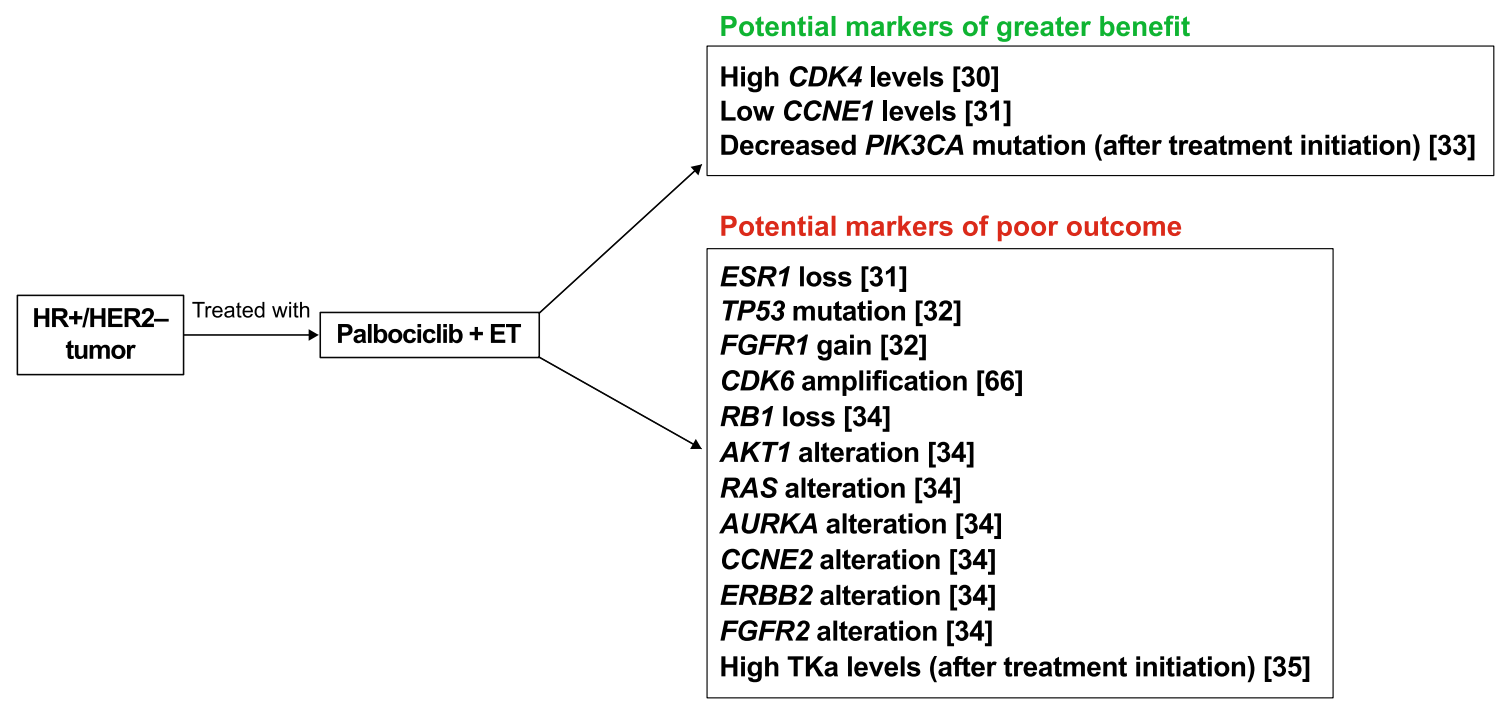

Fig. 2 Potential biomarkers predictive of response to palbociclib $[30-35,66]$. These markers have the potential to predict response to palbociclib in patients with HR+/HER2- breast cancer. ER estrogen receptor; $E T$ endocrine therapy; HER2- human epidermal growth fac-

inhibitors [34]. The study identified loss of $R B 1$; and alterations in $A K T 1, R A S, A U R K A, C C N E 2, E R B B 2$, and FGFR2 as potential CDK4/6 inhibitor resistance mechanisms [34]. A study also showed that an increase in plasma levels of thymidine kinase 1 activity (TKa) after 1 cycle of palbociclib treatment was associated with shorter median PFS, highlighting TKa levels as a predictor of early resistance to CDK4/6 inhibition [35]. Further research is warranted to confirm whether previously identified potential genomic biomarkers are predictive of response to CDK4/6 inhibitors [36].

\section{Palbociclib clinical trial data in Asian and Japanese patients}

Subgroup analyses of Asian patients enrolled in PALOMA-2 and PALOMA-3 showed a favorable efficacy and pharmacokinetic and safety profile generally consistent with that of the overall population. Ninety-five Asian patients (14.3\% of the overall population), including 46 Japanese patients, were enrolled in PALOMA-2, and 105 Asian patients (20.2\% of the overall population), including 35 Japanese patients, were enrolled in PALOMA-3 [37-39]. Among Asian patients in PALOMA-2, median PFS was 25.7 months with palbociclib plus letrozole versus 13.9 months with placebo plus letrozole (hazard ratio, 0.49 [95\% CI, 0.27-0.87]; $P=0.007$ ) [37]. Compared with non-Asians, Asians had higher geometric mean palbociclib $C_{\text {trough }}$ values (93.8 vs $61.7 \mathrm{ng} /$ $\mathrm{mL}$ ), but variability was lower (percent coefficient of variance: $32.3 \%$ vs $59.1 \%$ ), and the distribution of $C_{\text {trough }}$ values was generally similar [37]. In PALOMA-3, among Asian tor receptor 2-negative; $H R+$ hormone receptor-positive; $P I K 3 C A$ phosphatidylinositol-4,5-bisphosphate 3-kinase catalytic subunit alpha; $T K a$ thymidine kinase 1 activity

patients, median PFS was not reached in the palbociclib group and was 5.8 months in the placebo group (hazard ratio, 0.485 [95\% CI, 0.27-0.87]; $P=0.0065$ ) [39]. Asian and non-Asian patients had similar palbociclib exposure, with similar within-patient geometric mean steady-state palbociclib trough concentration values $(85.7$ and $74.8 \mathrm{ng} / \mathrm{mL}$, respectively) [39]. In both studies, neutropenia was the most common any-grade AE among Asian patients who received palbociclib, and was also more frequent among Asian than non-Asian patients [37, 39]. However, palbociclib discontinuation rates due to AEs among Asian and non-Asian patients were comparable (PALOMA-2: $10.8 \%$ vs 9.5\%; PALOMA-3: 0\% vs 5.1\%), suggesting that palbociclib AEs are manageable in Asian patients.

Subgroup analyses specifically looking at the Japanese cohorts from PALOMA-2 and PALOMA-3 were also conducted (Table 2). A total of 46 Japanese patients with $\mathrm{ER}+/ \mathrm{HER} 2-\mathrm{ABC}$ were enrolled in PALOMA-2; 32 patients received palbociclib plus letrozole and 14 received placebo plus letrozole [38]. Median PFS among Japanese patients was longer with palbociclib compared with placebo (22.2 vs 13.8 months; $P=0.103$ ), and PFS was not affected by dose reduction [38]. At the time of this report, OS data were not yet mature. Compared with that in non-Asians, the steadystate geometric mean palbociclib $C_{\text {trough }}$ value was higher in Japanese patients ( $95.4 \mathrm{vs} 61.7 \mathrm{ng} / \mathrm{mL}$ ), but the individual values were within a similar range. The incidence of anygrade hematologic AEs observed in the palbociclib group was higher among Japanese patients than that observed in the overall population of PALOMA-2 (Table 2). Overall, grade 3/4 AEs were observed more frequently among 


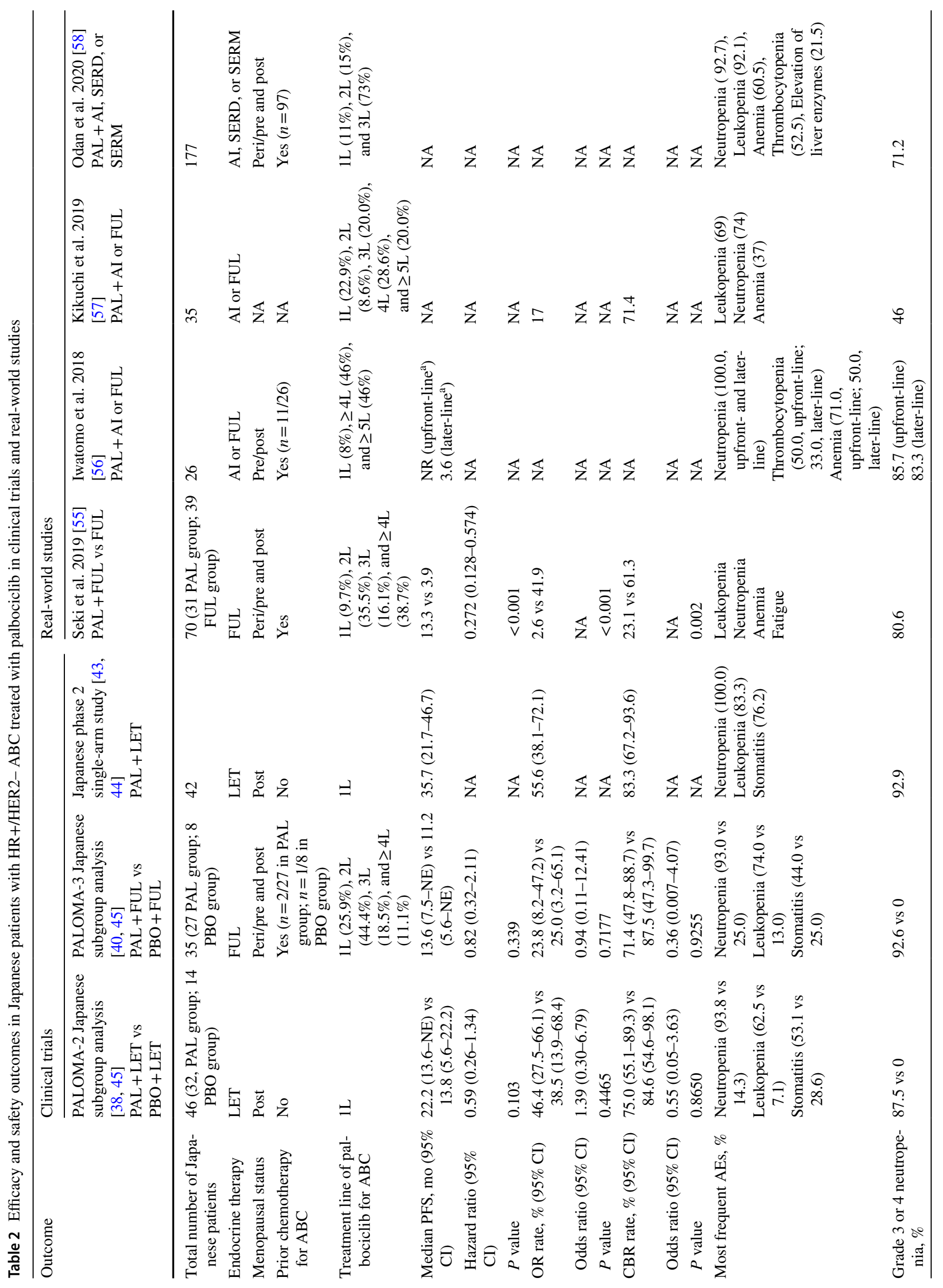




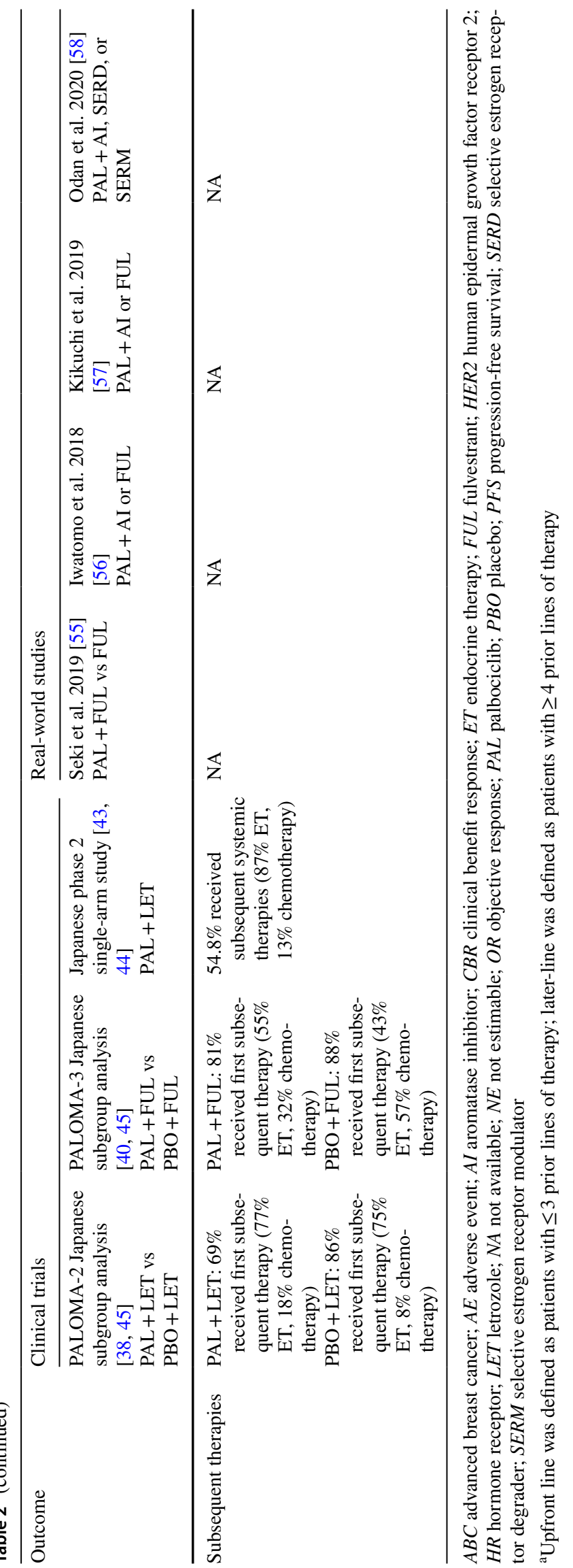


Japanese patients treated with palbociclib than those in the overall population. For example, neutropenia was observed in $87.5 \%$ of palbociclib-treated Japanese patients versus $66.4 \%$ of patients in the overall population. More Japanese patients experienced a dose reduction due to an AE compared with the overall population; neutropenia was associated with dose reduction in $31.3 \%$ of patients [38]. Overall, neutropenia was manageable with dose modification, and only $9.4 \%$ of patients discontinued study treatment because of neutropenia.

PALOMA-3 enrolled 35 Japanese patients with HR+/HER2- MBC whose disease had progressed on previous endocrine therapy; 27 patients received palbociclib plus fulvestrant and 8 patients received placebo plus fulvestrant [40]. Median PFS was longer among Japanese patients who received palbociclib versus those who received placebo (13.6 vs 11.2 months; $P=0.339$ ). Japanese and nonAsian patients had similar within-patient geometric mean $C_{\text {trough }}$ palbociclib levels at steady state $(84.4$ and $74.8 \mathrm{ng} /$ $\mathrm{mL}$, respectively), showing similar palbociclib exposure. Similar to the overall population, neutropenia was the most common AE with palbociclib treatment among Japanese patients; albeit, a higher rate of neutropenia was observed in Japanese patients versus the overall population (93.0\% vs $79.0 \%$ ). Febrile neutropenia was reported in 1 Japanese patient receiving palbociclib plus fulvestrant. Although more Japanese patients experienced a palbociclib dose reduction due to hematologic AEs (33\% due to neutropenia) than in the overall population, no Japanese patient discontinued palbociclib treatment because of AEs.

In a pooled analysis of Japanese patients from PALOMA-2, PALOMA-3, and the single-arm Japanese phase 2 study ( $n=101), 98.0 \%$ of Japanese patients who received palbociclib experienced all-grade neutropenia [41]; grade 3 or 4 neutropenia was reported in $90.1 \%$ of patients, but was manageable with dose modifications. Compared with Japanese patients who required cycle delay or dose interruption, patients who completed a $3 / 1$ schedule during the first 2 cycles with palbociclib had higher baseline neutrophil counts. In PALOMA- 2 and the Japanese phase 2 study, baseline neutrophil levels were positively correlated with neutrophil count at Cycle 1 Day 15. Importantly, exposure-response analyses in the overall populations in PALOMA-2 and PALOMA-3 showed similar PFS in patients with and without palbociclib dose reductions [42], and palbociclib dose reduction did not affect tumor response in Japanese patients [41]. In addition, no apparent correlation was observed between the post-treatment absolute neutrophil count and $C_{\text {trough }}$ in this pooled analysis or in PALOMA-2 or PALOMA-3, suggesting that the pharmacokinetics of palbociclib do not affect the incidence of neutropenia [38, 40, 41].

An open-label, single-arm, Japanese phase 2 study also examined the efficacy of palbociclib plus letrozole among postmenopausal patients with ER+/HER2- ABC who had no prior systemic anticancer therapy for $\mathrm{ABC}[43,44]$. A total of 42 patients received palbociclib plus letrozole and were included in efficacy analyses. In an updated analysis of the primary study [44], the 1-year PFS probability was $75.6 \%$, and the median PFS was 35.7 months. All 42 Japanese patients experienced any-grade neutropenia. Grade 3 or 4 neutropenia was observed in $92.9 \%$ of patients, and treatment-related febrile neutropenia (grade 3 ) was observed in 1 patient. In general, palbociclib-related AEs observed in the Japanese phase 2 study were managed through dose modification without affecting treatment duration or efficacy. Among the 23 patients (54.8\%) who received $\geq 1$ subsequent anticancer therapy, the majority received endocrine therapy $(87.0 \%)$ followed by chemotherapy (13.0\%). Similar to the Japanese phase 2 study, a recent report on subsequent treatment patterns after palbociclib plus endocrine therapy or placebo plus endocrine therapy in Japanese patients enrolled in PALOMA-2 and PALOMA-3 showed that endocrine therapy was the most common first subsequent therapy; chemotherapy was the second most comment subsequent therapy [45].

\section{Treatment with palbociclib in the real-world setting}

\section{Real-world evidence regarding treatment with palbociclib}

Palbociclib treatment in the real-world setting has been assessed in several retrospective studies and further support the efficacy and safety of palbociclib plus endocrine therapy for HR+/HER2- ABC. Table 1 summarizes real-world data from palbociclib studies that included more than 50 patients who were pre/peri- or postmenopausal women or men with HR+/HER2 - ABC. The Ibrance Real World Insights (IRIS) study used medical chart review data to evaluate palbociclib treatment in patients with confirmed HR+/HER2- ABC who received palbociclib in combination with either an aromatase inhibitor or fulvestrant in the United States, Argentina, and Germany [46, 47]. In addition, other real-world studies have published data from the United States, Italy, and Ireland [48-52]. In summary, real-world PFS was 15.1-26.4 months in the first-line setting [49-51], and 12.3-12.8 months in the second-line setting $[49,51]$, indicating that real-world efficacy with palbociclib combination treatment complements that observed in randomized controlled trials (PFS: PALOMA-2, 27.6 months in the first-line setting [23]; PALOMA-3, 11.2 months in the second- or later-line setting [24]). Similar to PALOMA-2 and PALOMA-3, commonly reported AEs in real-world studies included neutropenia, other hematologic AEs, and fatigue [48, 49, 52]. Of note, 
interstitial lung disease was also observed with CDK4/6 inhibitors in the real-world setting as well as PALOMA studies [27, 53]. Additionally, real-world data were utilized to expand the approved indications of palbociclib to include male patients with ABC in 2019 [54].

In Japan, the real-world efficacy of palbociclib was analyzed in 4 retrospective studies (Table 2) [55-58]. Whereas the sample sizes of Japanese real-world studies were small, the efficacy and safety results seem to be consistent with global real-world data. Findings from clinical trials and real-world data in Japanese patients showed that AEs associated with palbociclib therapy, including neutropenia, are managed effectively by dose modifications. However, further studies are needed to confirm the clinical effect of palbociclib in Japan, such as on efficacy, survival, AEs, and cost-effectiveness.

\section{Discussion}

CDK4/6 inhibitors have been shown to prolong PFS and OS as first-line or second-line treatment in patients with $\mathrm{ABC}$ [19, 20, 23-25, 59, 60]. Several reports in both the clinical trial and real-world settings have shown that the magnitude of PFS benefit is greater when palbociclib is used as an early-line therapy rather than in later-line settings, suggesting a limited clinical benefit among patients who receive it as a later-line option. First, hormone sensitivity decreases with subsequent endocrine therapy treatments, resulting in a reduced clinical benefit rate [61]. Findings from PALOMA-3 showed that among patients with sensitivity to previous endocrine therapy, median OS was 10 months longer with palbociclib plus fulvestrant versus placebo plus fulvestrant (hazard ratio for death, 0.72) [25]. Second, time to first subsequent chemotherapy with palbociclib treatment in PALOMA-2 was longer than that observed in PALOMA-3 (40.4 vs 17.6 months), suggesting quality of life was maintained for a longer period of time before chemotherapy was initiated [23, 25]. Finally, objective response with palbociclib treatment in PALOMA-2 was higher than that in PALOMA-3 among patients with measurable disease $(55.3 \%$ vs $25.0 \%)[16,62]$. It has also been shown that first-line treatment response was a key predictor of post-recurrence survival in patients with HR+/HER2- breast cancer [63]. Poor responses to first-line treatment were associated with unfavorable prognostic outcomes [63]. Success of first-line treatment may result in a positive and long-term relationship between doctor and patient. Together, these results suggest that in patients with $\mathrm{ABC}$ or $\mathrm{MBC}$, the optimal treatment option should be prescribed first-line.

Elucidating biomarkers that are predictive of palbociclib treatment benefit may highlight the optimal clinical application of this CDK4/6 inhibitor in patients with $\mathrm{ABC}$, including in those who are endocrine therapy-naive. Current evidence suggests that plasma thymidine kinase activity may predict response to palbociclib [35, 64]. Biomarkers such as this will be especially helpful to identify patients who will derive the greatest benefit from palbociclib combination therapy, including identifying patients sensitive to endocrine therapy who derive greater benefit from palbociclib. Moreover, it will be important to determine if such biomarkers are also predictive of treatment benefit in Asian and Japanese patients. In the event biomarkers identified in the overall population are not predictive in Asian patients, additional biomarker analyses in this population will be warranted.

Although AEs (e.g., neutropenia) are the main cause of palbociclib dose modification, analyses have suggested that there is no difference in efficacy between patients who did or did not experience a dose reduction [41]. Additionally, exposure-response analyses have shown that palbociclib dose reductions do not affect PFS [42]. Thus, AEs can be managed via dose modification without affecting the PFS benefit provided by palbociclib combination therapy.

Currently, several clinical trials of palbociclib for $\mathrm{HR}+/ \mathrm{HER} 2-\mathrm{ABC}$ are ongoing to address remaining clinical questions. First, it is not clear whether the optimal benefit of palbociclib can be achieved through first- or second-line treatment. The SONIA trial (ClinicalTrials.gov Identifier: NCT03425838) is currently evaluating whether the sequence of an aromatase inhibitor plus CDK4/6 inhibitor as firstline therapy, followed by fulvestrant as second-line therapy, is more effective than an aromatase inhibitor as first-line therapy followed by fulvestrant plus a CDK4/6 inhibitor as second-line therapy. Second, additional research on the choice of endocrine partner for CDK4/6 inhibitor combination therapy is also needed, as some clinical studies have already investigated the treatment benefit of using tamoxifen or an aromatase inhibitor with ribociclib [65]. Moreover, novel oral selective estrogen receptor degraders in combination with palbociclib are currently being evaluated (ClinicalTrials.gov Identifier: NCT03455270, NCT04711252).

Additionally, evaluation of the choice of subsequent therapy after disease progression while receiving palbociclib or after discontinuation of palbociclib due to an AE is warranted. The clinical study MAINTAIN is currently assessing the efficacy of ribociclib in patients whose disease progressed while receiving a CDK4/6 inhibitor (ClinicalTrials. gov Identifier: NCT02632045). A further understanding of the mechanisms of resistance to CDK4/6 inhibitors would aid in the assessment of subsequent treatment patterns. Current evidence suggests that the upregulation of various genes, such as CDK6 or CCNE1, may lead to resistance [66, 67]. Furthermore, real-time monitoring of tumor biology by ctDNA, as was demonstrated in PALOMA-3 [32, 33], may be a reasonable option for selecting optimal therapy depending on tumor characteristics (e.g., detection of the PIK3CA mutation would result in the selection of a PI3K inhibitor, such as 
buparlis or alpelisib [68]). The PADA-1 trial (ClinicalTrials. gov Identifier: NCT03079011), which is monitoring ctDNA for the occurrence of an ESR1 mutation in patients with ER+/HER2- MBC receiving palbociclib plus an aromatase inhibitor, may also be helpful in identifying the optimal subsequent therapy [69]. This type of personalized medicine is expected in the near future.

Finally, it is essential to understand which patients will achieve an OS benefit from a CDK4/6 inhibitor. For instance, there are three types of patients that can be identified via the Kaplan-Meier plots of PALOMA-2 and PALOMA-3 studies: (1) an early resistance group who have disease progression within approximately 6 months of treatment, (2) patients who experience disease progression near the median PFS time, and (3) patients who achieve a PFS benefit longer than the median PFS $[16,17,23,24]$. Thus far, there is minimal evidence to identify which patients will have longer survival with CDK4/6 inhibitor treatment, highlighting the importance of identifying a biomarker for this population. In addition, strategies to extend OS in patient groups 1 and 2 mentioned previously are warranted.

As was reviewed in this manuscript, several real-world studies demonstrate the efficacy and safety of palbociclib in clinical practice; however, there is still a lack of information available to answer the clinical questions discussed. Additional prospective clinical research studies and translational research studies are essential to help clarify these clinical questions associated with CDK4/6 inhibitor treatment.

\section{Conclusion}

Early-line palbociclib treatment of patients with $\mathrm{HR}+/$ HER2- ABC provides clinical benefit regardless of patient ethnicity. Many clinical trial and real-world studies have highlighted the prolonged PFS afforded by palbociclib combination therapy compared with endocrine therapy alone when used as a first-line treatment in patients with $\mathrm{HR}+$ /HER2 $-\mathrm{ABC}$, in the overall population and in subgroups of Asian and Japanese patients. The safety profile of palbociclib therapy, especially neutropenia, is manageable through dose modification without affecting treatment duration or efficacy both in clinical trials and real-world studies. Analyses suggest potential biomarkers could be predictive of response to CDK4/6 inhibitors (e.g., CDK4, CCNE1 levels). Further clinical research on biomarkers is merited to help improve outcomes in patients with HR+/HER2- ABC treated with $\mathrm{CDK} 4 / 6$ inhibitors as personalized medicine.

Acknowledgements Editorial support was provided by Jill Shults, $\mathrm{PhD}$, and Anny Wu, PharmD, of ICON plc (North Wales, PA, USA) and was funded by Pfizer Inc.

Funding This article was sponsored by Pfizer Inc.

\section{Declarations}

Conflict of interest The authors declare that they have no conflict of interest.

Disclosures N Masuda has received honoraria from AstraZeneca, Chugai, Eisai, Eli Lilly, Pfizer Inc, and Takeda and research funding from AstraZeneca, Chugai, Eisai, Eli Lilly, Daiichi Sankyo, Kyowa-Kirin, MSD, Novartis, and Pfizer Inc; H Iwata has received honoraria and research funding from AstraZeneca and Pfizer Inc and fees for promotional materials from AstraZeneca; $M$ Toi has received honoraria from AstraZeneca, Chugai, Eisai, Eli Lilly, Genomic Health Institute, Kyowa-Hakko Kirin, MSD, Novartis, Pfizer Inc, Taiho, and Takeda; research funding from AstraZeneca, Chugai, Eli Lilly, Novartis, Pfizer Inc, and Taiho; served as a consultant/independent contractor for Kyowa-Hakko Kirin; and served on the advisory board for Genomic Health Institute; N Kosaka is an employee of Pfizer Inc.

Open Access This article is licensed under a Creative Commons Attribution 4.0 International License, which permits use, sharing, adaptation, distribution and reproduction in any medium or format, as long as you give appropriate credit to the original author(s) and the source, provide a link to the Creative Commons licence, and indicate if changes were made. The images or other third party material in this article are included in the article's Creative Commons licence, unless indicated otherwise in a credit line to the material. If material is not included in the article's Creative Commons licence and your intended use is not permitted by statutory regulation or exceeds the permitted use, you will need to obtain permission directly from the copyright holder. To view a copy of this licence, visit http://creativecommons.org/licenses/by/4.0/.

\section{References}

1. Bray F, Ferlay J, Soerjomataram I et al (2018) Global cancer statistics 2018: GLOBOCAN estimates of incidence and mortality worldwide for 36 cancers in 185 countries. CA Cancer J Clin 68(6):394-424

2. Nakamura K, Okada E, Ukawa S et al (2017) Characteristics and prognosis of Japanese female breast cancer patients: the BioBank Japan project. J Epidemiol 27(S3):S58-S64

3. American Cancer Society. Breast Cancer Facts \& Figures 20192020. American Cancer Society, Inc. Available at: https://www. cancer.org/content/dam/cancer-org/research/cancer-facts-and-stati stics/breast-cancer-facts-and-figures/breast-cancer-facts-and-figur es-2019-2020.pdf. Accessed February 17, 2020

4. Giordano SH (2018) Breast cancer in men. N Engl J Med 378(24):2311-2320

5. Ellis MJ, Llombart-Cussac A, Feltl D et al (2015) Fulvestrant $500 \mathrm{mg}$ versus anastrozole $1 \mathrm{mg}$ for the first-line treatment of advanced breast cancer: overall survival analysis from the phase II FIRST study. J Clin Oncol 33(32):3781-3787

6. Iwata H, Masuda N, Ohno S et al (2013) A randomized, doubleblind, controlled study of exemestane versus anastrozole for the first-line treatment of postmenopausal Japanese women with hormone-receptor-positive advanced breast cancer. Breast Cancer Res Treat 139(2):441-451

7. Rugo H, Rumble B, Macrae E et al (2016) Endocrine therapy for hormone receptor-positive metastatic breast cancer: American Society of Clinical Oncology guideline. J Clin Oncol 34(25):3069-3103

8. Meyerson M, Harlow E (1994) Identification of G1 kinase activity for cdk6, a novel cyclin D partner. Mol Cell Biol 14(3):2077-2086 
9. Finn RS, Aleshin A, Slamon DJ (2016) Targeting the cyclindependent kinases (CDK) 4/6 in estrogen receptor-positive breast cancers. Breast Cancer Res 18(1):17

10. IBRANCE ${ }^{\circledR}$ capsules (palbociclib) (2019) Full prescribing information. Pfizer Inc, New York, NY

11. Pharmaceutical and Medical Devices Agency (PMDA). New drugs approved in September 2017. Available at: https://www. pmda.go.jp/english/review-services/reviews/approved-infor mation/drugs/0002.html. Accessed July 16, 2019

12. Shimoi T, Nagai SE, Yoshinami T et al (2020) The Japanese Breast Cancer Society Clinical Practice Guidelines for systemic treatment of breast cancer, 2018 edition. Breast Cancer. 27(3):322-331

13. Fry DW, Harvey PJ, Keller PR et al (2004) Specific inhibition of cyclin-dependent kinase 4/6 by PD 0332991 and associated antitumor activity in human tumor xenografts. Mol Cancer Ther 3(11): $1427-1438$

14. Toogood PL, Harvey PJ, Repine JT et al (2005) Discovery of a potent and selective inhibitor of cyclin-dependent kinase 4/6. J Med Chem 48(7):2388-2406

15. Iwata $H$ (2018) Clinical development of CDK4/6 inhibitor for breast cancer. Breast Cancer 25(4):402-406

16. Finn RS, Martin M, Rugo HS et al (2016) Palbociclib and letrozole in advanced breast cancer. $\mathrm{N}$ Engl J Med 375(20):1925-1936

17. Turner NC, Ro J, Andre F et al (2015) Palbociclib in hormone-receptor-positive advanced breast cancer. N Engl J Med 373(3):209-219

18. Slamon DJ, Neven P, Chia S et al (2018) Phase III randomized study of ribociclib and fulvestrant in hormone receptor-positive, human epidermal growth factor receptor 2-negative advanced breast cancer: MONALEESA-3. J Clin Oncol 36(24):2465-2472

19. Hortobagyi GN, Stemmer SM, Burris HA et al (2016) Ribociclib as first-line therapy for HR-positive, advanced breast cancer. $\mathrm{N}$ Engl J Med 375(18):1738-1748

20. Sledge GW Jr, Toi M, Neven P et al (2017) MONARCH 2: abemaciclib in combination with fulvestrant in women with $\mathrm{HR}+$ / HER2- advanced breast cancer who had progressed while receiving endocrine therapy. J Clin Oncol 35(25):2875-2884

21. Goetz MP, Toi M, Campone M et al (2017) MONARCH 3: abemaciclib as initial therapy for advanced breast cancer. J Clin Oncol 35(32):3638-3646

22. Marra A, Curigliano G (2019) Are all cyclin-dependent kinases 4/6 inhibitors created equal? NPJ Breast Cancer 5:27

23. Rugo HS, Finn RS, Dieras V et al (2019) Palbociclib plus letrozole as first-line therapy in estrogen receptor-positive/human epidermal growth factor receptor 2-negative advanced breast cancer with extended follow-up. Breast Cancer Res Treat 174(3):719-729

24. Cristofanilli M, DeMichele A, Giorgetti C et al (2018) Predictors of prolonged benefit from palbociclib plus fulvestrant in women with endocrine-resistant hormone receptor-positive/human epidermal growth factor receptor 2-negative metastatic breast cancer in PALOMA-3. Eur J Cancer 104:21-31

25. Turner NC, Slamon DJ, Ro J et al (2018) Overall survival with palbociclib and fulvestrant in advanced breast cancer. N Engl J Med 379(20):1926-1936

26. Turner NC, Finn RS, Martin M et al (2018) Clinical considerations of the role of palbociclib in the management of advanced breast cancer patients with and without visceral metastases. Ann Oncol 29(3):669-680

27. Finn RS, Rugo HS, Gelmon KA et al (2021) Long-term pooled safety analysis of palbociclib in combination with endocrine therapy for hormone receptor-positive/human epidermal growth factor receptor 2-negative advanced breast cancer: updated analysis with up to 5 years of follow-up. Oncologist 26(5):e749-e755
28. Harbeck N, Iyer S, Turner N et al (2016) Quality of life with palbociclib plus fulvestrant in previously treated hormone receptorpositive, HER2-negative metastatic breast cancer: patient-reported outcomes from the PALOMA-3 trial. Ann Oncol 27(6):1047-1054

29. Rugo HS, Dieras V, Gelmon KA et al (2018) Impact of palbociclib plus letrozole on patient-reported health-related quality of life: results from the PALOMA-2 trial. Ann Oncol 29(4):888-894

30. Finn RS, Liu Y, Zhu Z et al (2020) Biomarker analyses of response to cyclin-dependent kinase 4/6 inhibition and endocrine therapy in women with treatment-naive metastatic breast cancer. Clin Cancer Res 26(1):110-121

31. Turner NC, Liu Y, Zhu Z et al (2019) Cyclin E1 expression and palbociclib efficacy in previously treated hormone receptor-positive metastatic breast cancer. J Clin Oncol 37(14):1169-1178

32. O'Leary B, Cutts RJ, Huang X et al (2021) Circulating tumor DNA markers for early progression on fulvestrant with or without palbociclib in ER+ advanced breast cancer. J Natl Cancer Inst 113:309-317

33. O'Leary B, Hrebien S, Morden JP et al (2018) Early circulating tumor DNA dynamics and clonal selection with palbociclib and fulvestrant for breast cancer. Nat Commun 9(1):896

34. Wander SA, Cohen O, Gong X et al (2020) The genomic landscape of intrinsic and acquired resistance to cyclin-dependent kinase 4/6 inhibitors in patients with hormone receptor-positive metastatic breast cancer. Cancer Discov 10(8):1174-1193

35. McCartney A, Bonechi M, De Luca F et al (2020) Plasma thymidine kinase activity as a biomarker in patients with luminal metastatic breast cancer treated with palbociclib within the TREnd trial. Clin Cancer Res 26(9):2131-2139

36. Gao JJ, Cheng J, Bloomquist E et al (2020) CDK4/6 inhibitor treatment for patients with hormone receptor-positive, HER2-negative, advanced or metastatic breast cancer: a US Food and Drug Administration pooled analysis. Lancet Oncol 21(2):250-260

37. Im SA, Mukai H, Park IH et al (2019) Palbociclib plus letrozole as first-line therapy in postmenopausal Asian women with metastatic breast cancer: results from the phase III, randomized PALOMA-2 study. J Glob Oncol 5:1-19

38. Mukai H, Shimizu C, Masuda N et al (2019) Palbociclib in combination with letrozole in patients with estrogen receptor-positive, human epidermal growth factor receptor 2-negative advanced breast cancer: PALOMA-2 subgroup analysis of Japanese patients. Int J Clin Oncol 24:274-287

39. Iwata H, Im S-A, Im Y-H et al (2017) PALOMA-3: phase III trial of fulvestrant with or without palbociclib in premenopausal and postmenopausal women with hormone receptor-positive, human epidermal growth factor receptor 2-negative metastatic breast cancer that progressed on prior endocrine therapy-safety and efficacy in Asian patients. J Glob Oncol 3(4):289-303

40. Masuda N, Inoue K, Nakamura R et al (2019) Palbociclib in combination with fulvestrant in patients with hormone receptor-positive, human epidermal growth factor receptor 2-negative advanced breast cancer: PALOMA-3 subgroup analysis of Japanese patients. Int J Clin Oncol 24:262-273

41. Masuda N, Mukai H, Inoue K et al (2019) Neutropenia management with palbociclib in Japanese patients with advanced breast cancer. Breast Cancer 26(5):637-650

42. Zheng J, Yu Y, Durairaj C et al (2021) Impact of dose reduction on efficacy: implications of exposure-response analysis of palbociclib. Target Oncol. 16(1):69-76

43. Masuda N, Nishimura R, Takahashi M et al (2018) Palbociclib in combination with letrozole as first-line treatment for advanced breast cancer: a Japanese phase II study. Cancer Sci 109(3):803-813

44. Takahashi M, Masuda N, Nishimura R et al (2020) Palbociclibletrozole as first-line treatment for advanced breast cancer: 
updated results from a Japanese phase 2 study. Cancer Med. 9(14):4929-4940

45. Masuda N, Mukai H, Inoue K et al (2021) Analysis of subsequent therapy in Japanese patients with hormone receptor-positive/human epidermal growth factor receptor 2-negative advanced breast cancer who received palbociclib plus endocrine therapy in PALOMA-2 and -3. Breast Cancer 28(2):335-345

46. Taylor-Stokes G, Mitra D, Waller J et al (2019) Treatment patterns and clinical outcomes among patients receiving palbociclib in combination with an aromatase inhibitor or fulvestrant for $\mathrm{HR}+/$ HER2-negative advanced/metastatic breast cancer in real-world settings in the US: results from the IRIS study. Breast 43:22-27

47. Waller J, Mitra D, Mycock K et al (2019) Real-world treatment patterns and clinical outcomes in patients receiving palbociclib for hormone receptor-positive, human epidermal growth factor receptor 2-negative advanced or metastatic breast cancer in Argentina: the IRIS study. J Glob Oncol. 5:JGO1800239

48. Pizzuti L, Giordano A, Michelotti A et al (2018) Palbociclib plus endocrine therapy in HER2 negative, hormonal receptor-positive, advanced breast cancer: a real-world experience. J Cell Physiol 234(6):7708-7717

49. Varella L, Eziokwu AS, Jia X et al (2019) Real-world clinical outcomes and toxicity in metastatic breast cancer patients treated with palbociclib and endocrine therapy. Breast Cancer Res Treat 176(2):429-434

50. Wilkie J, Schickli MA, Berger MJ et al (2019) Progression-free survival for real-world use of palbociclib in hormone receptorpositive metastatic breast cancer. Clin Breast Cancer 20(1):33-40

51. Xi J, Oza A, Thomas S et al (2019) Retrospective analysis of treatment patterns and effectiveness of palbociclib and subsequent regimens in metastatic breast cancer. J Natl Compr Canc Netw 17(2):141-147

52. Watson GA, Deac O, Aslam R et al (2019) Real-world experience of palbociclib-induced adverse events and compliance with complete blood count monitoring in women with hormone receptor-positive/HER2-negative metastatic breast cancer. Clin Breast Cancer 19(1):e186-e194

53. Raschi E, Fusaroli M, Ardizzoni A et al (2021) Cyclin-dependent kinase 4/6 inhibitors and interstitial lung disease in the FDA adverse event reporting system: a pharmacovigilance assessment. Breast Cancer Res Treat 186:219-227

54. Wedam S, Fashoyin-Aje L, Bloomquist E et al (2019) FDA approval summary: palbociclib for male patients with metastatic breast cancer. Clin Cancer Res 26(6):1208-1212

55. Seki H, Sakurai T, Maeda Y et al (2019) Efficacy and safety of palbociclib and fulvestrant in Japanese patients with ER+/HER2advanced/metastatic breast cancer. In Vivo 33(6):2037-2044

56. Iwamoto N, Aruga T, Kuroi K et al (2018) Efficacy and toxicity of palbociclib in heavily pretreated patients with metastatic breast cancer. Ann Cancer Res Ther 26(2):105-109

57. Kikuchi M, Tanaka Y, Yokota M et al (2019) Analysis of the selection of CDK4/6 inhibitors based on experience using palbociclib. Biomed Rep 11(6):253-256

58. Odan N, Kikawa Y, Matsumoto H et al (2020) Real-world outcomes of treating advanced breast cancer patients with palbociclib: a multicenter retrospective cohort study in Japan-the KBCOG-14 study. Breast Cancer (Auckl) 14:1178223420983843

59. Slamon DJ, Neven P, Chia S et al (2020) Overall survival with ribociclib plus fulvestrant in advanced breast cancer. N Engl J Med 382(6):514-524

60. Sledge GW Jr, Toi M, Neven P et al (2020) The effect of abemaciclib plus fulvestrant on overall survival in hormone receptor-positive, ERBB2-negative breast cancer that progressed on endocrine therapyMONARCH 2: a randomized clinical trial. JAMA Oncol 6(1):116-124

61. Iwase H, Yamamoto Y (2015) Clinical benefit of sequential use of endocrine therapies for metastatic breast cancer. Int J Clin Oncol 20(2):253-261
62. Cristofanilli M, Turner NC, Bondarenko I et al (2016) Fulvestrant plus palbociclib versus fulvestrant plus placebo for treatment of hormone-receptor-positive, HER2-negative metastatic breast cancer that progressed on previous endocrine therapy (PALOMA-3): final analysis of the multicentre, double-blind, phase 3 randomised controlled trial. Lancet Oncol 17(4):425-439

63. Yamamura J, Kamigaki S, Tsujie M et al (2019) Response to firstline recurrence treatment influences survival in hormone receptor-positive, HER2-negative breast cancer: a multicenter study. In Vivo 33(1):281-287

64. Bagegni N, Thomas S, Liu N et al (2017) Serum thymidine kinase 1 activity as a pharmacodynamic marker of cyclin-dependent kinase 4/6 inhibition in patients with early-stage breast cancer receiving neoadjuvant palbociclib. Breast Cancer Res 19(1):123

65. Bardia A, Campos-Gomez S, Hurvitz SA, Lu Y, Im S, Franke F et al (2018) Tamoxifen (TAM) or a non-steroidal aromatase inhibitor (NSAI) with ribociclib (RIB) in premenopausal patients (pts) with hormone receptor-positive (HR+), HER2-negative (HER2-) advanced breast cancer (ABC): MONALEESA-7 subgroup analysis [abstract]. Ann Oncol 29(suppl 8):VIII106-VIII107

66. Yang $\mathrm{C}, \mathrm{Li} \mathrm{Z}$, Bhatt $\mathrm{T}$ et al (2017) Acquired CDK6 amplification promotes breast cancer resistance to CDK4/6 inhibitors and loss of ER signaling and dependence. Oncogene 36(16):2255-2264

67. Herrera-Abreu MT, Palafox M, Asghar U et al (2016) Early adaptation and acquired resistance to CDK4/6 inhibition in estrogen receptor-positive breast cancer. Cancer Res 76(8):2301-2313

68. André F, Ciruelos E, Rubovszky G et al (2019) Alpelisib for PIK3CA-mutated, hormone receptor-positive advanced breast cancer. N Engl J Med 380(20):1929-1940

69. Clement Bidard F, Callens C, Dalenc F et al (2020) Prognostic impact of ESR1 mutations in ER+ HER2- MBC patients prior treated with first line $\mathrm{AI}$ and palbociclib: an exploratory analysis of the PADA-1 trial. J Clin Oncol 38(suppl):abstract 1010

70. Matsushime H, Ewen ME, Strom DK et al (1992) Identification and properties of an atypical catalytic subunit (p34PSK-J3/cdk4) for mammalian D type G1 cyclins. Cell 71(2):323-334

71. Caldon CE, Daly RJ, Sutherland RL et al (2006) Cell cycle control in breast cancer cells. J Cell Biochem 97(2):261-274

72. Tripathy D, Bardia A, Sellers WR (2017) Ribociclib (LEE011): mechanism of action and clinical impact of this selective cyclindependent kinase 4/6 inhibitor in various solid tumors. Clin Cancer Res 23(13):3251-3262

73. VanArsdale T, Boshoff C, Arndt KT et al (2015) Molecular pathways: targeting the cyclin D-CDK4/6 axis for cancer treatment. Clin Cancer Res 21(13):2905-2910

74. Finn RS, Dering J, Conklin D et al (2009) PD 0332991, a selective cyclin D kinase 4/6 inhibitor, preferentially inhibits proliferation of luminal estrogen receptor-positive human breast cancer cell lines in vitro. Breast Cancer Res 11(5):R77

75. Alvarez-Fernandez M, Malumbres M (2020) Mechanisms of sensitivity and resistance to CDK4/6 inhibition. Cancer Cell 37(4):514-529

76. Goel S, DeCristo MJ, Watt AC et al (2017) CDK4/6 inhibition triggers anti-tumour immunity. Nature 548(7668):471-475

77. Watt AC, Cejas P, DeCristo MJ et al (2021) CDK4/6 inhibition reprograms the breast cancer enhancer landscape by stimulating AP-1 transcriptional activity. Nat Cancer 2(1):34-48

78. Klein ME, Kovatcheva M, Davis LE et al (2018) CDK4/6 inhibitors: the mechanism of action may not be as simple as once thought. Cancer Cell 34(1):9-20

Publisher's Note Springer Nature remains neutral with regard to jurisdictional claims in published maps and institutional affiliations. 